OPEN ACCESS

Edited by:

Keiichi Okazaki,

Niigata University, Japan

Reviewed by:

Pengfang Zhu,

Shenyang Agricultural University,

China

Zhansheng Li, Institute of Vegetables and Flowers,

Chinese Academy of Agricultural Sciences (CAAS), China

*Correspondence:

Xiao-Wei Zhang xiaowei5737@163.com

Yuxiang Yuan

yuxiangyuan126@126.com

tThese authors have contributed equally to this work and share first

authorship

Specialty section: This article was submitted to Plant Breeding, a section of the journal

Frontiers in Plant Science

Received: 22 December 2021

Accepted: 27 January 2022

Published: 17 February 2022

Citation:

Yang S, Liu H, Zhao Y, Su H, Wei X Wang $Z$, Zhao $X$, Zhang $X$-W and Yuan Y (2022) Map-Based Cloning and Characterization of Br-dyp 1, a Gene Conferring Dark Yellow Petal Color Trait in Chinese Cabbage (Brassica rapa L. ssp. pekinensis).

Front. Plant Sci. 13:841328. doi: 10.3389/fp/s.2022.841328

\section{Map-Based Cloning and} Characterization of Br-dyp1, a Gene Conferring Dark Yellow Petal Color Trait in Chinese Cabbage (Brassica rapa L. ssp. pekinensis)

\author{
Shuangjuan Yang ${ }^{1,2 t}$, Honglei Liu' ${ }^{1,2 \dagger}$, Yanyan Zhao ${ }^{1 \dagger}$, Henan Su', Xiaochun Wei', \\ Zhiyong Wang ${ }^{1}$, Xiaobin Zhao', Xiao-Wei Zhang ${ }^{1,2 *}$ and Yuxiang Yuan ${ }^{1,2 *}$

\begin{abstract}
IInstitute of Horticulture, Henan Academy of Agricultural Sciences, Zhengzhou, China, ${ }^{2}$ School of Agricultural Sciences,
\end{abstract} \\ Zhengzhou University, Zhengzhou, China
}

Flower color is an important trait in Brassica species. However, genes responsible for the dark yellow flower trait in Chinese cabbage have not been reported. In this study, we identified a dark-yellow-flowered Chinese cabbage line SD369. Genetic analysis indicated that the dark yellow flower trait in SD369 was controlled by a single recessive locus, Br-dyp1 (dark yellow petal color 1 in Brassica rapa). Using bulked segregant RNA sequencing and kompetitive allele-specific PCR assays, Br-dyp1 was fine-mapped to an interval of $53.6 \mathrm{~kb}$ on chromosome A09. Functional annotation analysis, expression analysis, and sequence variation analysis revealed that Bra037130 (BraA09.ZEP), which encodes a zeaxanthin epoxidase, was the most likely candidate gene for Br-dyp1. Carotenoid profile analysis suggested that Bra037130 (BraA09.ZEP) might participate in the epoxidation from zeaxanthin to violaxanthin. The $679 \mathrm{bp}$ insertion in dark yellow petal caused premature stop codon, thus caused the loss-of-function of the enzyme zeaxanthin epoxidase (ZEP), which disturbed the carotenoid metabolism, and caused the increased accumulation of total carotenoid, and finally converted the flower color from yellow to dark yellow. Comparative transcriptome analysis also showed that the "carotenoid biosynthesis" pathway was significantly enriched, and genes involved in carotenoid degradation and abscisic acid biosynthesis and metabolism were significantly downregulated. Furthermore, we developed and validated the functional marker Br-dyp1InDel for Br-dyp1. Overall, these results provide insight into the molecular basis of carotenoid-based flower coloration in B. rapa and reveal valuable information for markerassisted selection breeding in Chinese cabbage.

Keywords: Brassica rapa, flower color, dark yellow petal color, fine-mapping, breeding 


\section{INTRODUCTION}

Flower color is one of the most important traits in Brassica species and is particularly useful for ornamental and landscaping purposes (Zhang et al., 2018a; Liu et al., 2020b). In breeding, flower color can be used to evaluate variety purity in hybrid production (Zhang et al., 2018b; Yang et al., 2021). Brassica flowers are usually yellow, but can also be white, milky white, orange, or dark yellow (Zhang et al., 2018a). The gene carotenoid cleavage dioxygenase 4 (CCD4) underlies the white flower trait in Brassica napus and Brassica oleracea (Zhang et al., 2015; Han et al., 2019). In Brassica rapa, the orange flower color as well as the orange coloration of the inner leaves is controlled by the carotenoid isomerase (BrCRTISO) gene (Su et al., 2014). Our previous study showed that the BrWF3 gene, which encodes a diacylglycerol acyltransferase and is homologous to PES2 in Arabidopsis, controls the white flower trait in Chinese cabbage (B. rapa L. ssp. pekinensis; Yang et al., 2021). However, the gene underlying the dark yellow flower trait in Chinese cabbage or in $B$. rapa species has not been reported.

Carotenoids are a group of more than 700 lipid-soluble pigments synthesized in plastids (Gonzalez-Jorge et al., 2016; Li et al., 2016). In chloroplasts, carotenoids are essential structural and functional components of the antenna complex of the photosynthesis system, with roles in light harvesting, non-photochemical quenching, and limiting membrane damage by reactive oxygen species and singlet oxygen species (Walter and Strack, 2011; Zhang et al., 2015; Gonzalez-Jorge et al., 2016). Carotenoids in chromoplasts endow flowers and fruits with different colors, such as orange, yellow, and red, which attract animals for pollination and seed dispersal (Kevan and Baker, 1983). Carotenoids are also biosynthetic precursors for the synthesis of the plant hormones abscisic acid (ABA) and strigolactone (Frey et al., 2006; Alder et al., 2012).

The enzyme zeaxanthin epoxidase (ZEP) plays a critical role in carotenoid biosynthesis, which is responsible for the epoxidation of zeaxanthin to yield antheraxanthin and subsequently violaxanthin. Violaxanthin can be deepoxidated to antheraxanthin and then zeaxanthin by the enzyme violaxanthin de-epoxidase. This reversible epoxidation/ deepoxidation is referred to as the xanthophyll cycle, in which deepoxidation to zeaxanthin is favored under high-light conditions while epoxidation to violaxanthin predominates in moderate-light conditions. The rapid formation of zeaxanthin via the xanthophyll cycle is indispensable for the dissipation of excess energy by non-photochemical quenching, while violaxanthin is a precursor for ABA biosynthesis; thus, xanthophyll cycle is one of the critical processes contributing to plant fitness and stress tolerance (Alboresi et al., 2011; Gao et al., 2013; Gonzalez-Jorge et al., 2016; Lou et al., 2017). Many studies have showed that ZEP proteins are involved in $\mathrm{ABA}$ biosynthesis, $Z E P$ mutants display low $\mathrm{ABA}$ levels and almost no ABA upregulation under drought stress, while the overexpression of $Z E P$ enhances tolerance to osmotic stress, which suggests that the ZEP enzyme plays a critical role in the ABA-mediated stress response (Marin et al., 1996; Agrawal et al., 2001; Xiong et al., 2002; Park et al., 2008).
However, the impact of ZEP on carotenoid pigmentation in the flowers of Chinese cabbage has not been investigated.

In this study, we conducted positional cloning of the dark yellow petal color gene $(B r-d y p 1)$ in Chinese cabbage by using $\mathrm{F}_{2}$ populations, which derived from the dark-yellow-flowered inbred line "SD369" and the yellow-flowered DH line "R1611." Furthermore, we conducted carotenoid profile analysis and comparative transcriptome analysis to figure out the mechanisms underlying the dark yellow flower color pigmentation. In addition, we developed and validated a functional marker. This work will promote marker-assisted selection breeding and the exploration of molecular mechanisms that regulate flower color variation in Chinese cabbage or in B. rapa.

\section{MATERIALS AND METHODS}

\section{Plant Materials}

The yellow-petaled double haploid (DH) line R16-11 $\left(\mathrm{P}_{1}\right)$ and the dark-yellow-petaled inbred Chinese cabbage line SD369 $\left(\mathrm{P}_{2}\right)$ were used as parents to generate $\mathrm{F}_{1}, \mathrm{~F}_{2}, \mathrm{BC}_{1} \mathrm{P}_{1}$, and $\mathrm{BC}_{1} \mathrm{P}_{2}$ populations for the inheritance and mapping studies. The $\mathrm{BC}_{1} \mathrm{P}_{1}$ and $\mathrm{BC}_{1} \mathrm{P}_{2}$ populations were created by backcrossing $\mathrm{F}_{1}$ plants with $\mathrm{R} 16-11$ or SD369, respectively. The petal color trait was investigated visually at the flowering stage. Statistical analyses of the segregation ratios of the $\mathrm{F}_{2}$ and $\mathrm{BC}_{1} \mathrm{P}_{1}$ populations were carried out through chi-square test $\left(\chi^{2}\right)$. Additionally, eight yellow-petaled materials, eight dark-yellow-petaled materials, eight orange-petaled materials, and eight white-petaled materials were used to analyze mutations in the candidate gene (Supplementary Table 1). All the materials used in this study were provided by the Institute of Horticulture, Henan Academy of Agricultural Sciences.

\section{Carotenoids Identification and Quantification}

Carotenoids composition was measured by MetWare ${ }^{1}$ based on the AB Sciex QTRAP 6500 LC-MS/MS platform. Petals from 10 dark-yellow-petaled $F_{2}$ plants were combined to form one replicate (referred to as the DY-bulk), and petals from 10 yellow-petaled $\mathrm{F}_{2}$ plants were included in the Y-bulk. In total, three replicates were assessed. Fresh petals were freezedried, ground into powder $(30 \mathrm{~Hz}, 1.5 \mathrm{~min})$, and stored at $-80^{\circ} \mathrm{C}$ until needed. For each sample, $50 \mathrm{mg}$ powder was weighted and extracted with $0.5 \mathrm{ml}$ of a mixed solution of n-hexane: acetone: ethanol $(1: 1: 1, \mathrm{v} / \mathrm{v} / \mathrm{v})$ with $0.01 \% \mathrm{BHT}(\mathrm{g} / \mathrm{ml})$, and $10 \mu \mathrm{l}$ of $(13 \mathrm{C} 10)-\beta$-carotene solution $(20 \mu \mathrm{g} / \mathrm{ml})$ were added into the extract as internal standards for quantification. The extract was vortexed for $20 \mathrm{~min}$ at room temperature. The supernatants were collected after centrifugation at $12000 \mathrm{r} / \mathrm{min}$ for $5 \mathrm{~min}$ at $4^{\circ} \mathrm{C}$. The residue was re-extracted by repeating the above steps again under the same conditions. Saturated sodium chloride solution $(0.5 \mathrm{ml})$ was added to the supernatant, after which the mixture was vortexed, and the upper layer

${ }^{1}$ http://www.metware.cn/ 
was collected. This step was repeated two times more. Then, the supernatant was evaporated to dryness and dissolved in $0.5 \mathrm{ml}$ of MTBE, then $0.5 \mathrm{ml} 10 \% \mathrm{KOH}-\mathrm{MeOH}$ was added, the mixture was vortexed again, and the reaction was allowed to take place at room temperature overnight. After the reaction, $1 \mathrm{ml}$ of saturated sodium chloride solution and $0.5 \mathrm{ml}$ of MTBE were added, followed by vortexing, and the upper layer was collected. This step was repeated two times, and the supernatant was evaporated to dryness and reconstituted in $100 \mu$ l of mixed solution of $\mathrm{MeOH} / \mathrm{MTBE}(1: 1, \mathrm{v} / \mathrm{v})$. The solution was filtered through a $0.22 \mu \mathrm{m}$ membrane filter for further LC-MS/ MS analysis.

The sample extracts were analyzed using a UPLC-APCI-MS/ MS system (UPLC, ExionLC ${ }^{\mathrm{TM}}$ AD; MS, Applied Biosystems 6,500 Triple Quadrupole), which was performed as described in previous studies (Liu et al., 2020a; Wang et al., 2020; Zhou et al., 2020; Yang et al., 2021). The integrated peak area of each carotenoid detected in the samples was substituted into the linear equations of standard curves for content calculation; finally, the absolute content data for the carotenoids in the actual samples were obtained (Supplementary Table 2). The specific procedure for calculation of the carotenoid content was performed as Yang et al. (2021).

\section{Bulked Segregant RNA Sequencing and Analysis}

The DY-bulk and Y-bulk each with three replicates used for carotenoid analysis were also used for RNA sequencing. Six cDNA were constructed and sequenced at BioMarker Tech Co., Ltd. (Beijing, China). The Illumina HiSeq X 10 platform was used to generate 150-base paired-end reads for each library. To preliminarily map the candidate gene, the clean reads from the three DY-bulk replicates were merged to form a single read file, and another merged file was obtained from the three Y-bulk replicates. Then, the merged read files were aligned to the $B$. rapa reference genome (V1.5) using BWA software (Li and Durbin, 2010). The single-nucleotide polymorphism (SNP) and insertion/deletion (InDel) variants were called using SAMtools software (Li et al., 2009). The SNP index was calculated for all genomic positions in the DY-bulk and Y-bulk and the $\Delta$ (SNP index) was calculated by subtracting the SNP index of the Y-bulk from that of the DY-bulk, which was performed as previously described (Abe et al., 2012; Takagi et al., 2013; Yang et al., 2021). The candidate region of $B r-d y p 1$ was identified by sliding window analysis with a $1-\mathrm{Mb}$ width and a $50-\mathrm{kb}$ increment at the 95\% confidence level according to (Yang et al., 2021).

To identify differentially expressed genes (DEGs) between the DY-bulk and Y-bulk, the clean reads of each library were aligned to the B. rapa V1.5 reference genome using HISAT2 software with the default parameters (Kim et al., 2015). Then, the fragments per kilobase of transcript per million mapped reads value of each gene was calculated to estimate gene expression levels. DEGs were identified using the DESeq2 package (v1.6.3; Love et al., 2014). Genes with false discovery rate (FDR) $\leq 0.05$ and $\mid \log 2$ (fold change) $\mid \geq 1$ were recognized as DEGs. Gene Ontology (GO) enrichment analysis was carried out using the topGO package (v2.18.0; Alexa et al., 2010). Kyoto Encyclopedia of Genes and Genomes (KEGG) pathway enrichment analysis was implemented using KOBAS (v2.0) software (Mao et al., 2005; Wu et al., 2006).

\section{Kompetitive Allele-Specific PCR Marker and Linkage Map Development}

To validate the results of Bulked Segregant RNA Sequencing (BSR-Seq) and map the Br-dyp1 gene, we selected SNPs showing polymorphism between the two bulks and nearing the candidate region for Kompetitive Allele-Specific PCR (KASP) marker development. The detailed procedures for KASP marker development and KASP assays were performed as described by (Yang et al., 2020). The developed KASP markers were first screened between R16-11 and SD369. Then, polymorphic KASP markers (Supplementary Table 3) were employed to genotype the $\mathrm{F}_{2}$ population containing 94 individuals. The genetic linkage map was constructed using JoinMap 4.0 software (Van Ooijen, 2006). Recombination values were converted into genetic map distances (cM) following the Kosambi mapping function (Kosambi, 1943).

For the fine-mapping of the candidate gene, approximately $3,500 \mathrm{~F}_{2}$ seeds were planted, and 743 individuals with a dark yellow petal phenotype were used for screening more recombinants.

\section{Cloning and Sequence Analysis of the Candidate Genes}

We designed primer pairs according to the $B$. rapa reference genome to clone the DNA and cDNA sequence of the candidate gene. Phanta Flash Master Mix (Vazyme Biotech Co., Ltd., Nanjing, China) was deployed to amplify the candidate gene. The PCR products were sequenced by Sunya Biotech Co., Ltd. (Zhengzhou, China), and sequence alignments were performed using DNAMAN software. The coding sequences (CDSs) of candidate gene from R16-11 and SD369 were submitted to GenBank under the accession numbers OL436220 (R16-11) and OL436221 (SD369).

\section{Quantitative Real-Time PCR}

Total RNA was extracted from tissues of roots, stems, leaves, sepals, petals, stamens, and pistils from R16-11 and SD369. BrGAPDH was used as an internal control (Qi et al., 2010; Su et al., 2014). Quantitative Real-Time PCR (qRT-PCR) was performed with $2 \times$ TB Green Premix Ex Taq II (TaKaRa, Japan) on a Roche LightCycler 480-II System (Roche Applied Sciences, Beijing, China). Relative expression levels were calculated using the $2^{-\Delta \Delta C t}$ method (Livak and Schmittgen, 2001). The results from three biological replicates are shown.

\section{RESULTS}

\section{The Dark Yellow Petal Color Trait of SD369 Is Controlled by a Single Recessive Gene}

The phenotypic analysis showed significant differences in petal color between the two parental lines (Figure 1). In R16-11 $\left(\mathrm{P}_{1}\right)$, the petals showed stable yellow coloration at the flowering stage, whereas those of SD369 $\left(\mathrm{P}_{2}\right)$ exhibited dark yellow 

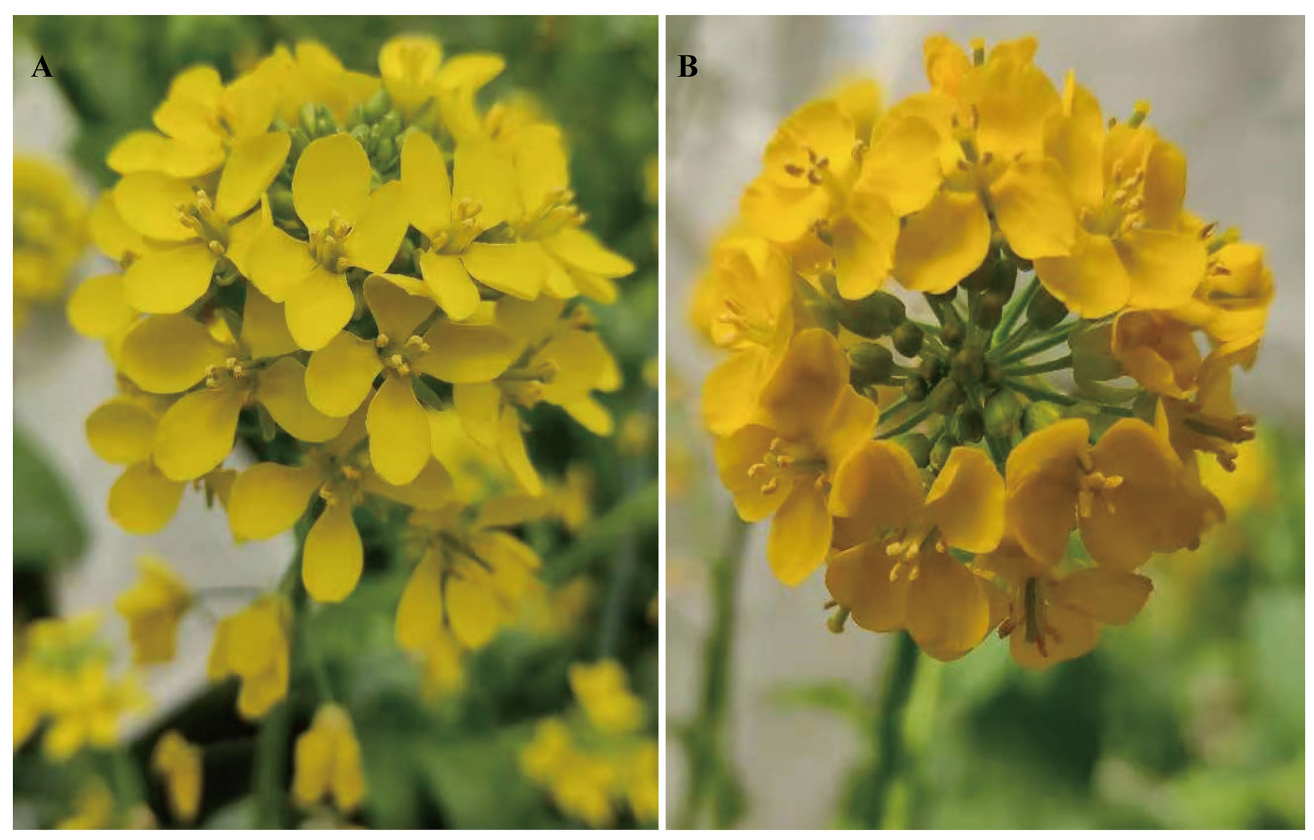

FIGURE 1 | Phenotypic characterization of flower color in the two parent lines (R16-11 and SD369). The flower color of R16-11 (A) is yellow at the flowering stage, while dark yellow for SD369 (B).

coloration (Figures 1A,B). All 15 individuals in the $F_{1}$ population showed a yellow petal phenotype, as did R16-11. Phenotypic segregation was observed in the $\mathrm{F}_{2}$ and $\mathrm{BC}_{1} \mathrm{P}_{2}$ populations, and the petals of the plants in these two populations exhibited two kinds of colorations, yellow or dark yellow, corresponding to the coloration of either R16-11 or SD369, respectively. In a small $F_{2}$ population, 176 plants exhibited yellow petals, and 54 showed dark yellow petals, corresponding to a segregation ratio of $3: 1$ by the chi-square test (Table 1). In a larger $F_{2}$ population, the segregation ratio was also 3:1 (2,371 yellow:743 dark yellow, $\chi^{2}=2.16$ ). The numbers of individuals with yellow and dark yellow petals in the $\mathrm{BC}_{1} \mathrm{P}_{2}$ population were 125 and 115, respectively, corresponding to a ratio of $1: 1$ according to the chi-square test $\left(\chi^{2}=0.42<\chi_{0.05}^{2}=3.84, p<0.05\right)$. Furthermore, all $200 \mathrm{BC}_{1} \mathrm{P}_{1}$ plants showed yellow petals. All of these results demonstrate that the dark yellow petal color trait of SD369 is controlled by a monogenic recessive gene (Table 1). We named this locus Br-dyp1 (dark yellow petal color 1 in B. rapa).

\section{The Carotenoid Profile Is Altered in Dark Yellow Petals}

The composition and content of carotenoids in petals of the Y-bulk and DY-bulk were determined using UPLC-APCI-MS/ MS system under saponification treatment. The results showed that the carotenoid profiles of the DY-bulk were quite different from those of the Y-bulk. Ten carotenoids composition were identified in Y-bulk and DY-bulk, including three carotenes and seven xanthophylls (Figure 2; Supplementary Table 4). The total content of carotenes was slightly lower in the DY-bulk than in the Y-bulk (Supplementary Table 4). The total content of xanthophylls accounted for approximately $92.0 \%$ or $98.9 \%$ of the total carotenoids in the Y-bulk and DY-bulk, respectively, and the contents of almost all xanthophylls were higher in the DY-bulk than in the Y-bulk. For example, the amounts of lutein, zeaxanthin, and antheraxanthin in the DY-bulk increased about 6.7-fold, 11.2-fold, and 11.5-fold than that in Y-bulk. However, the amount of violaxanthin decreased about 3.6-fold in DY-bulk (Figure 2; Supplementary Table 4). The total content of carotenoids showed a remarkable 4.7-fold increase in dark yellow petals relative to that in the yellow petals (Supplementary Table 4). Taken together, these findings suggested that the dark yellow petal phenotype in SD369 resulted from increased contents of carotenoids, particularly lutein, zeaxanthin, and antheraxanthin.

\section{Fine-Mapping of the Br-dyp1 Gene}

$B r-d y p 1$ was preliminarily mapped using BSR-seq. A total of $47,206,290,38,634,054$, and 54,612,412 clean reads were obtained for the three Y-bulks, while 47,676,208, 51,940,890, and 49,967,262 clean reads were obtained for the three DY-bulks (Supplementary Table 5). Reads from the three Y-bulks were merged as the Y-pool, and reads from the three DY-bulks were merged to form the DY-pool. The Y-pool and DY-pool clean reads were aligned to the $B$. rapa V1.5 genome, and a total of 348,456 SNPs and 29,092 InDels were identified between these two pools. The $\Delta$ (SNP index) of each position was calculated for sliding window analysis. According to the null hypothesis, a $2.7 \mathrm{Mb}$ region from 3.8 to $6.5 \mathrm{Mb}$ on chromosome A09 exhibiting significant linkage disequilibrium was identified as the candidate region for the dark yellow petal trait at a $99 \%$ confidence level 
TABLE 1 | Genetic analysis of the petal trait in parents and in crosses between R16-11 and SD369.

\begin{tabular}{|c|c|c|c|c|c|c|}
\hline Generations & Total & Yellow & Dark yellow & Expected ratio & $\chi^{2}$ & $x_{0.05}^{2}$ \\
\hline$P_{1}(\mathrm{R} 16-11)$ & 10 & 10 & 0 & - & - & - \\
\hline $\mathrm{P}_{2}$ (SD369) & 10 & 0 & 10 & - & - & - \\
\hline$F_{1}$ & 15 & 15 & 0 & - & - & - \\
\hline $\mathrm{F}_{2}$-small & 230 & 176 & 54 & $3: 1$ & 0.28 & 3.84 \\
\hline $\mathrm{F}_{2}$-large & 3,114 & 2,371 & 743 & $3: 1$ & 2.16 & 3.84 \\
\hline $\mathrm{BC}_{1} \mathrm{P}_{1}\left(\mathrm{~F}_{1} \times \mathrm{R} 16-11\right)$ & 200 & 200 & 0 & - & - & - \\
\hline $\mathrm{BC}_{1} \mathrm{P}_{2}\left(\mathrm{~F}_{1} \times \mathrm{SD} 369\right)$ & 240 & 125 & 115 & $1: 1$ & 0.42 & 3.84 \\
\hline
\end{tabular}

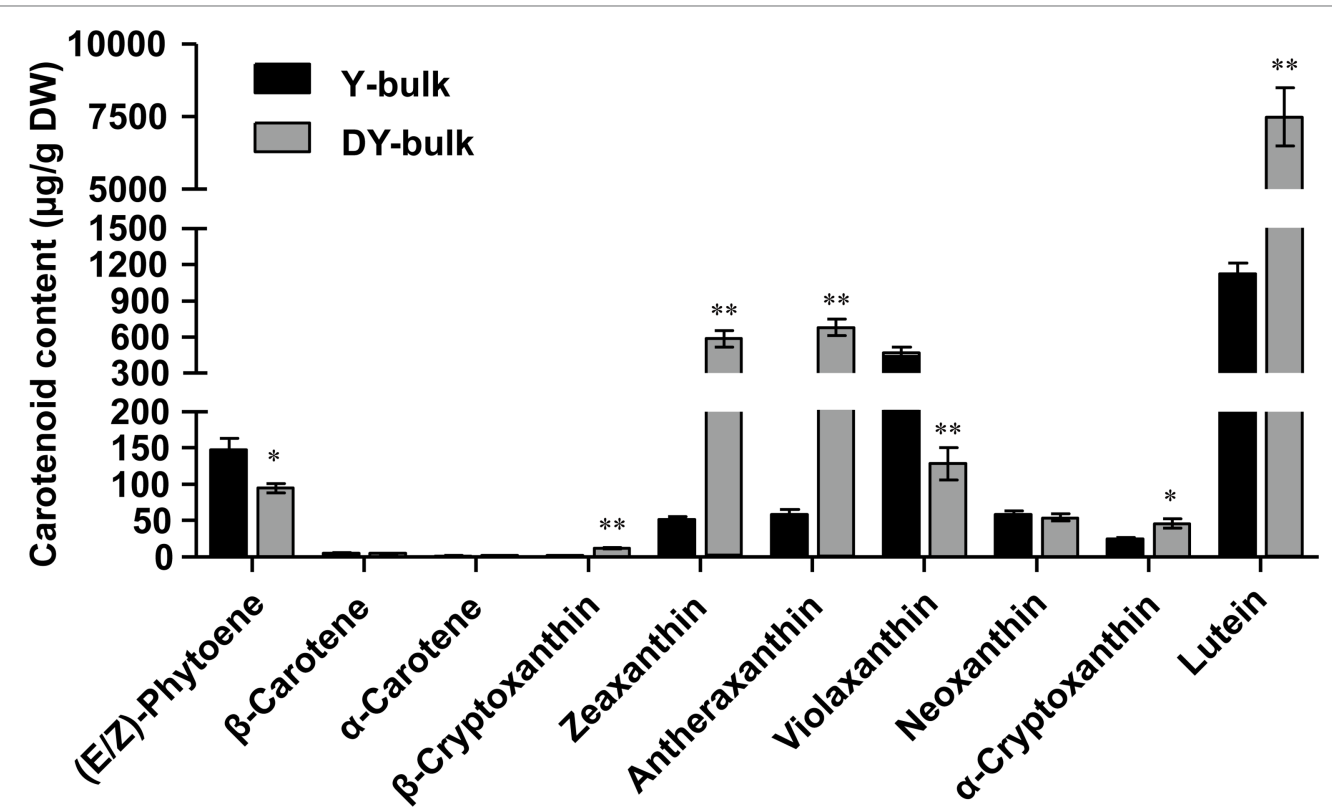

FIGURE 2 | Carotenoid composition in petals from Y-bulk and DY-bulk. Carotenoids extracts from yellow and dark yellow petals were subjected to UPLC-APCIMS/MS system under saponification treatment. Error bars indicate SE $(n=3)$. Value of $* p<0.05$ (Student's $t$-test). Value of $* * p<0.01$ (Student's $t$-test).

(Supplementary Figure 1; Supplementary Table 6), which was consistent with the genetic analysis showing the dark yellow petal color trait was controlled by a single recessive nuclear gene.

To validate the BSR-Seq results, 35 KASP markers in the candidate region were developed and used to screen the two parents. The results showed that 13 KASP markers (Supplementary Table 3) exhibited good polymorphism. These 13 markers were further genotyped in $94 \mathrm{~F}_{2}$ plants for linkage analysis (Supplementary Table 7). The results showed that the marker $\mathrm{SH}-\mathrm{K} 27$ co-segregated with the $B r-d y p 1$ gene in the preliminary mapping population (Figure 3A). There was one recombinant individual between $B r-d y p 1$ and $\mathrm{SH}-\mathrm{K} 25$ and $\mathrm{SH}-\mathrm{K} 29$. The genetic distances between the Br-dyp1 locus and $\mathrm{SH}-\mathrm{K} 25$ and $\mathrm{SH}-\mathrm{K} 29$ were 0.6 and $0.5 \mathrm{cM}$, respectively (Figure 3A). The order of the markers in the genetic map is consistent with that in the physical map (Figure 3A).

To fine-map the Br-dyp1 locus, we screened 743 dark-yellowpetaled $\mathrm{F}_{2}$ plants using the flanking markers SH-K34 and $\mathrm{SH}-\mathrm{K} 11$ and identified 54 recombinants. All the 54 recombinants were further genotyped using SH-K35, SH-K24, SH-K25, SH-K27, and $\mathrm{SH}-\mathrm{K} 29$, based on which 11 recombinants (type 4 and type 8) were identified (Figure 3B). Then, eight new markers were developed (Supplementary Table 3) and were further used to screen all the 11 recombinants using the KASP assay. The results delimited the $B r-d y p 1$ gene to a $53.6 \mathrm{~kb}$ interval between markers $\mathrm{SH}-\mathrm{K} 38$ and $\mathrm{SH}-\mathrm{K} 43$, each with two recombinants (type 5 and type 6; Figure 3B). Four markers, namely, SH-K41, SH-K36, SH-K37, and SH-K27, co-segregated with the $B r-d y p 1$ gene in the fine-mapping population (Figure 3B).

\section{Candidate Gene Analysis}

DNA sequences in the fine-mapping interval $(53.6 \mathrm{~kb})$ of $B r-d y p 1$ were analyzed according to the $B$. rapa reference genome. Totally, 11 annotated or predicted genes were identified in the mapping region (Table 2). Among them, Bra037130 (BraA09. $Z E P$ ), a homolog of $Z E P$ in Arabidopsis, could be the candidate gene (Table 2). ZEP encodes a zeaxanthin epoxidase that catalyzes the conversion of zeaxanthin to antheraxanthin and 


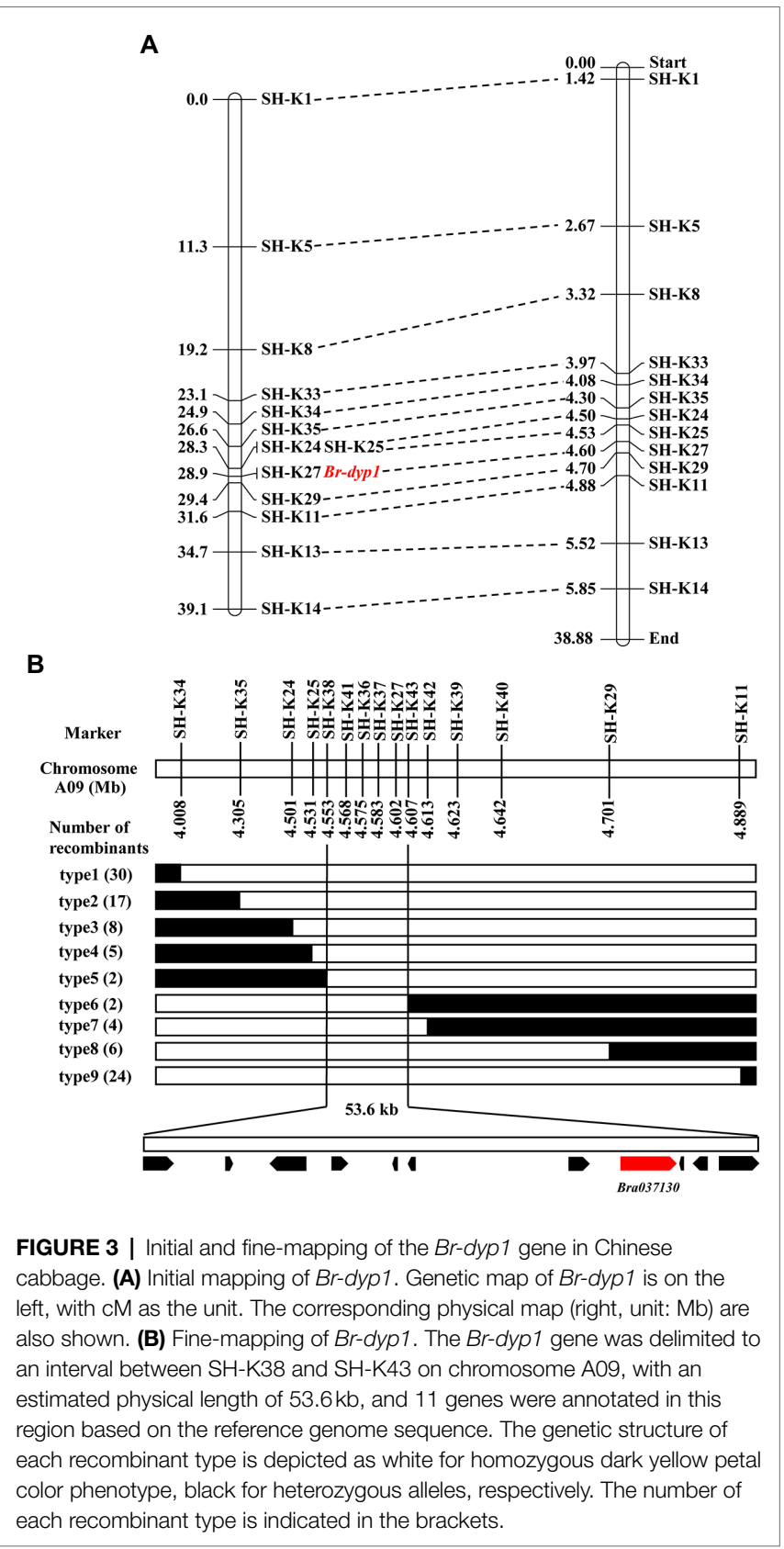

violaxanthin in the carotenoid biosynthesis pathway (Liu et al., 2020b).

Next, we examined the expression of the candidate gene Bra037130 (BraA09.ZEP) in different tissues of the two parent lines. qRT-PCR analysis using primer pairs Br-dyp1-qF1 and Br-dyp1-qR1 (Supplementary Table 8) revealed that the expression pattern of Bra037130 was significantly different between the parental lines. In any of the seven tissues that we examined, the expression of Bra037130 was much lower in the dark-yellow-petaled parent SD369 than in the yellowpetaled parent R16-11 (Figure 4A). The highest levels of Bra037130 (BraA09.ZEP) were present in the stamens and petals in both parental lines (Figure 4A).
TABLE 2 | Annotated genes in the candidate interval of the Br-dyp1 locus.

\begin{tabular}{|c|c|c|c|}
\hline Gene name & $\begin{array}{l}\text { Gene position } \\
\text { on A09 }\end{array}$ & $\begin{array}{l}\text { Arabidopsis } \\
\text { homolog }\end{array}$ & Gene function \\
\hline Bra037123 & $\begin{array}{c}4,553,464- \\
4,555,964\end{array}$ & АТЗG46730 & $\begin{array}{l}\text { NB-ARC domain- } \\
\text { containing disease } \\
\text { resistance protein }\end{array}$ \\
\hline Bra037124 & $\begin{array}{c}4,560,614- \\
4,561,153\end{array}$ & AT5G67190 & $\begin{array}{l}\text { Encodes a member } \\
\text { of the DREB } \\
\text { subfamily A-5 of } \\
\text { ERF/AP2 } \\
\text { transcription factor } \\
\text { family; DEAR2 }\end{array}$ \\
\hline Bra037125 & $\begin{array}{c}4,564,557- \\
4,567,632\end{array}$ & AT5G67170 & $\begin{array}{l}\text { SEC-C motif- } \\
\text { containing protein }\end{array}$ \\
\hline Bra037126 & $\begin{array}{c}4,569,807- \\
4,571,165\end{array}$ & AT5G67160 & $\begin{array}{l}\text { Encodes a member } \\
\text { of the BAHD } \\
\text { acyltransferase } \\
\text { superfamily; EPS1 }\end{array}$ \\
\hline Bra037127 & $\begin{array}{c}4,575,174- \\
4,575,557\end{array}$ & AT5G67070 & $\begin{array}{l}\text { Rapid Alkalinization } \\
\text { Factor; RALF34 }\end{array}$ \\
\hline Bra037128 & $\begin{array}{c}4,576,524- \\
4,577,141\end{array}$ & AT5G67060 & $\begin{array}{l}\text { Encodes a bHLH } \\
\text { transcription factor; } \\
\text { HEC1 }\end{array}$ \\
\hline Bra037129 & $\begin{array}{c}4,590,446- \\
4,592,182\end{array}$ & AT5G67050 & $\begin{array}{l}\text { alpha/beta- } \\
\text { Hydrolases } \\
\text { superfamily protein }\end{array}$ \\
\hline Bra037130 & $\begin{array}{c}4,594,979- \\
4,599,768\end{array}$ & AT5G67030 & $\begin{array}{l}\text { Zeaxanthin } \\
\text { epoxidase; ZEP }\end{array}$ \\
\hline Bra037131 & $\begin{array}{c}4,600,167- \\
4,600,430\end{array}$ & AT5G66985 & $\begin{array}{l}\text { Hypothetical protein; } \\
\text { HUP44 }\end{array}$ \\
\hline Bra037132 & $\begin{array}{c}4,601,342- \\
4,602,476\end{array}$ & AT5G66980 & $\begin{array}{l}\text { AP2/B3-like } \\
\text { transcriptional factor } \\
\text { family protein }\end{array}$ \\
\hline Bra037133 & $\begin{array}{c}4,603,501- \\
4,606,981\end{array}$ & AT5G66960 & $\begin{array}{l}\text { Prolyl oligopeptidase } \\
\text { family protein }\end{array}$ \\
\hline
\end{tabular}

To characterize the sequence of the candidate genes in the parental lines, the primer pair Br-dyp1-F and Br-dyp1-R2 (Supplementary Table 8) were designed. Sequence analysis indicated that the candidate gene of R16-11 was $3,020 \mathrm{bp}$ in length and contained 14 exons and 13 introns (Figure 5A; Supplementary Figure 2). The CDS of the candidate gene in R16-11 was $1965 \mathrm{bp}$ in length (Supplementary Figure 2). Sequence alignment showed that there were $43 \mathrm{SNP}$ variations and $11 \mathrm{InDel}$ variations between the genomic sequences of R16-11 and SD369 (Supplementary Table 9; Supplementary Figure 3). Among these variations, the most significant sequence variation was a $679 \mathrm{bp}$ insertion located at $240 \mathrm{bp}$ of the gDNA, within the first exon in SD369 (Figure 5A; Supplementary Table 10). The $679 \mathrm{bp}$ insertion caused a premature stop codon at the 93 a.a position (Figure 5B). Amino acid sequence alignment indicated that the deduced amino acid sequence of Bra037130 (BraA09.ZEP) in R16-11 was highly identical to the ZEP protein sequence in Arabidopsis (Supplementary Figure 4), and it contained four conserved motifs: two lipocalin conserved motifs (145-162 a.a and 264-283 a.a), a long monooxygenase domain (223-429 a.a), and a Forkhead-associated domain (579-625 a.a; Supplementary Figure 4). The 679 bp insertion in BraA09.ZEP caused the loss of the four conserved domains 

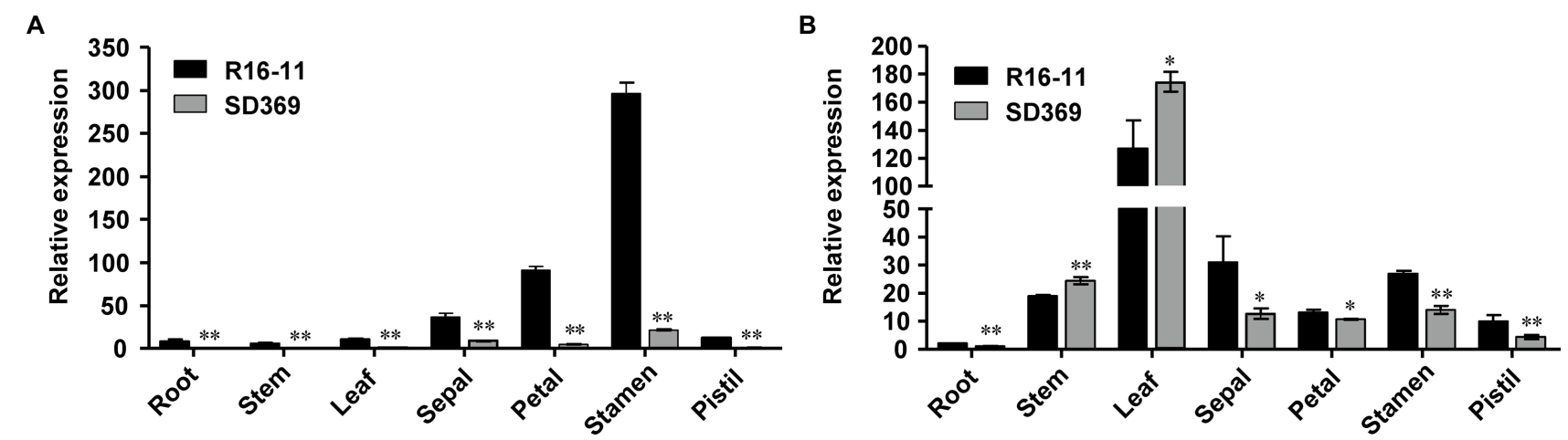

FIGURE 4 | Gene expression data analysis. Quantitative RT-PCR of Br-dyp1 (Bra037130; A) and BraA07.ZEP (Bra012127; B) in different tissues of the two parents. The BrGAPDH were used as an internal control. Error bars indicate SE $(n=3)$. Value of $* p<0.05$ (Student's $t$-test). Value of $* * p<0.01$ (Student's $t$-test).

and ultimately resulted in the loss of function of the ZEP protein in dark yellow petals.

Based on the $679 \mathrm{bp}$ insertion in dark-yellow-petaled parent SD369, a functional marker Br-dyp1-InDel (primers pair Br-dyp1ful-F and Br-dyp1-sp-R1; Supplementary Table 8), which could amplify a 1,644-bp and 965-bp product from line SD369 and R16-11, respectively, were developed and were assayed in different materials. The results showed that Br-dyp1-InDel co-segregated with the petal color phenotype in the $\mathrm{F}_{2}$ population (Figure 5C). Furthermore, eight yellow-petaled materials, eight dark yellow-petaled materials, eight orange-petaled materials, and eight white-petaled materials were genotyped using Br-dyp1InDel. As expected, all the eight dark yellow-petaled materials showed the same genotype as SD369, and all eight yellowpetaled materials showed the same genotype as R16-11 (Figure 5D). Interestingly, the eight orange-petaled and eight white-petaled materials also exhibited the same genotype as R16-11 (Figure 5E), which implied that the genes controlling the orange and white petal color trait were different from BraA09.ZEP and the 679bp insertion only existed in darkyellow-petaled materials. Overall, these findings suggest that the Bra037130 (BraA09.ZEP) gene is the most promising candidate gene for the dark yellow petal color gene $B r-d y p 1$ in Chinese cabbage.

\section{The Coding Sequence and Expression Pattern of BraA07.ZEP Show Only a Little Difference Between Dark Yellow and Yellow Petals}

Given that the coding sequence of BraA07.ZEP (Bra012127) was very similar to that of $B r-d y p 1$ ( $B r a A 09 . Z E P$ ), with $87.02 \%$ identity, we designed a primer pair, ZEP-A07-ful-F and ZEP-A07ful-R (Supplementary Table 8), to amplify the full-length CDS of BraA07.ZEP in R16-11 and SD369. The CDS of BraA07. ZEP from R16-11 and SD369 were submitted to GenBank under the accession numbers OL436222 (R16-11) and OL436223 (SD369). Sequence alignment revealed that there were 29 SNP variations between the coding sequences of R16-11 and SD369 (Supplementary Table 10; Supplementary Figure 5).
Among the 29 SNPs, 27 SNPs were synonymous mutations, and only 2 SNPs caused non-synonymous mutations, which did not affect the protein function (Supplementary Table 10; Supplementary Figure 6). The expression pattern of BraA07. $Z E P$ in the parental lines was also checked using primer pair ZEP-A07-qF and ZEP-A07-qR. The results revealed that the highest transcript levels were detected in the leaves, whereas low levels were found in petals and other tissues (Figure 4B). Furthermore, the expression of BraA07.ZEP in the petals of R16-11 was only slightly higher than that in SD369 (Figure 4B), which indicated a functional divergence between BraA07.ZEP and Br-dyp1 (BraA09.ZEP).

\section{Transcriptome Analysis in Dark Yellow and Yellow Petals}

To examine the global effect of the Br-dyp1 (BraA09.ZEP) mutation on gene expression in Chinese cabbage, RNA-seq analysis was employed to profile gene expression differences in petals from the Y-bulk and DY-bulk, each with three replicates. Approximately 290 million clean reads were generated for the six samples and $83.9-87.5 \%$ were uniquely mapped to the $B$. rapa (Chiifu-401) reference genome (Supplementary Table 5). All the clean reads were deposited in the NCBI Short Read Archive database under accession number PRJNA779176. Statistical analysis identified 835 DEGs with at least two-fold changes between the Y-bulk and DY-bulk from the three biological replicates (FDR $\leq 0.05)$. Among these DEGs, 248 genes were upregulated and 587 genes were downregulated in the dark yellow petals.

GO enrichment analysis of the 587 downregulated genes revealed that most DEGs were assigned to the "chloroplast stroma (GO: 0009570)" and "chloroplast thylakoid membrane (GO: 0009535)" terms in the cellular component category (Figure 6A), which was compatible with the ZEP localization in chloroplasts (Rock et al., 1992). Zeaxanthin is involved in non-photochemical quenching (NPQ) and thylakoid stacking, and thus affects the PSII function (Rock et al., 1992). Additionally, zeaxanthin serves important functions as an antioxidant in the lipid phase of the membrane and is likely to act as a key 

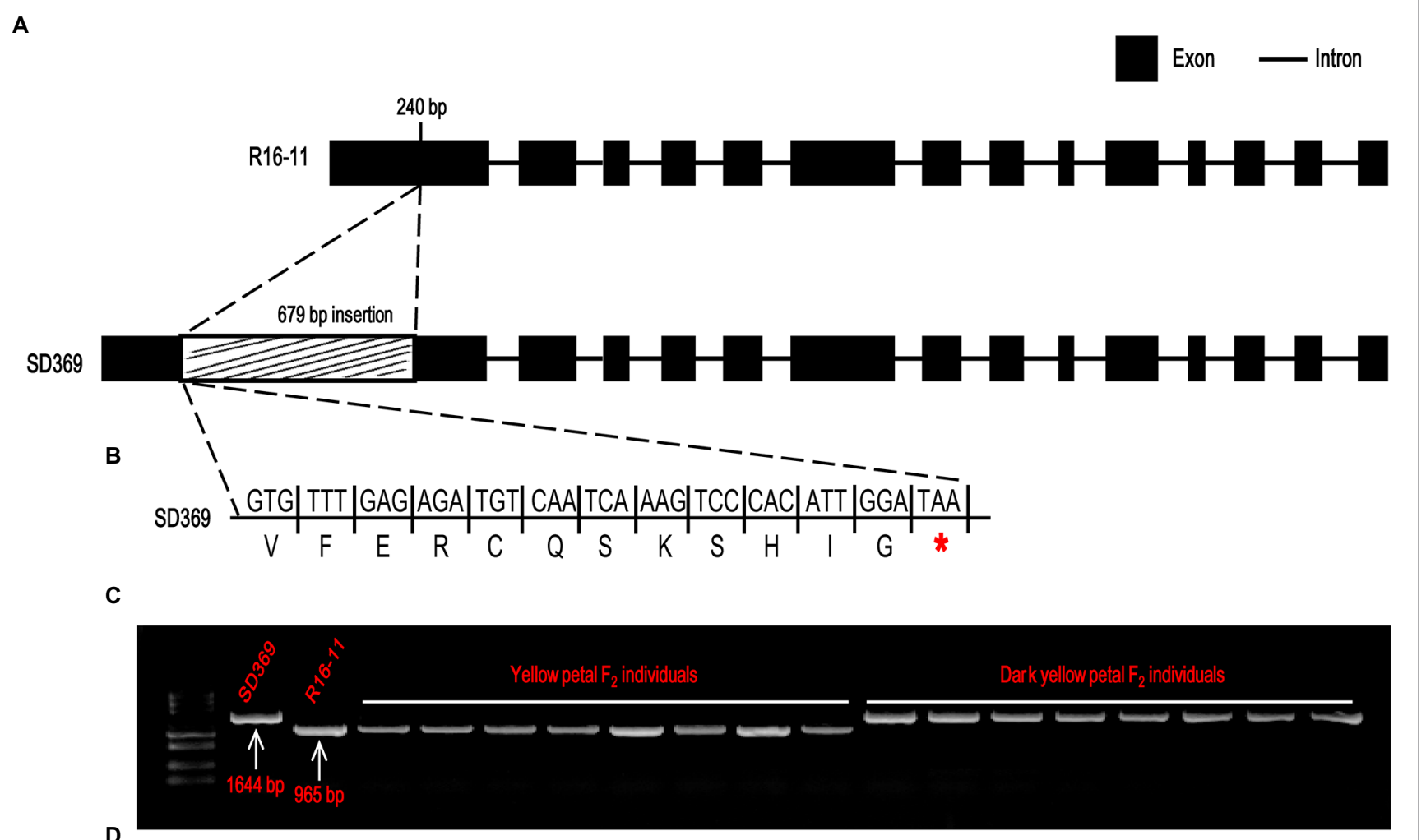

D

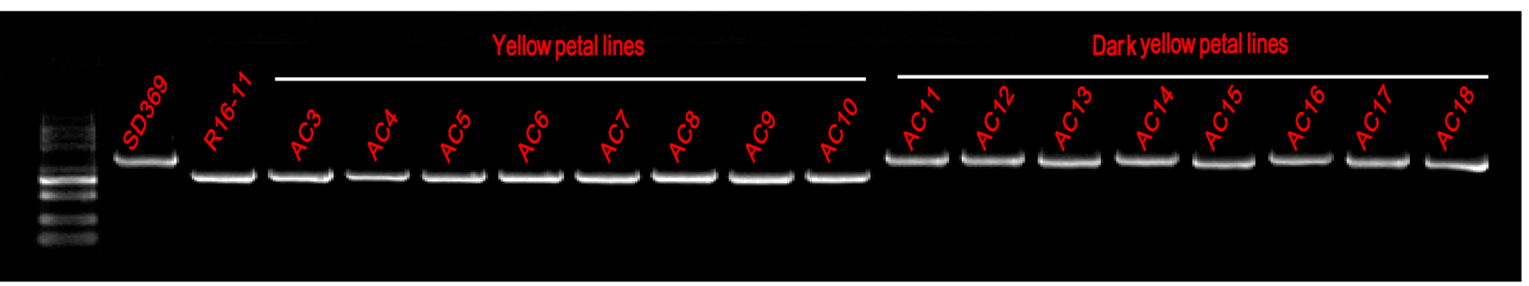

E

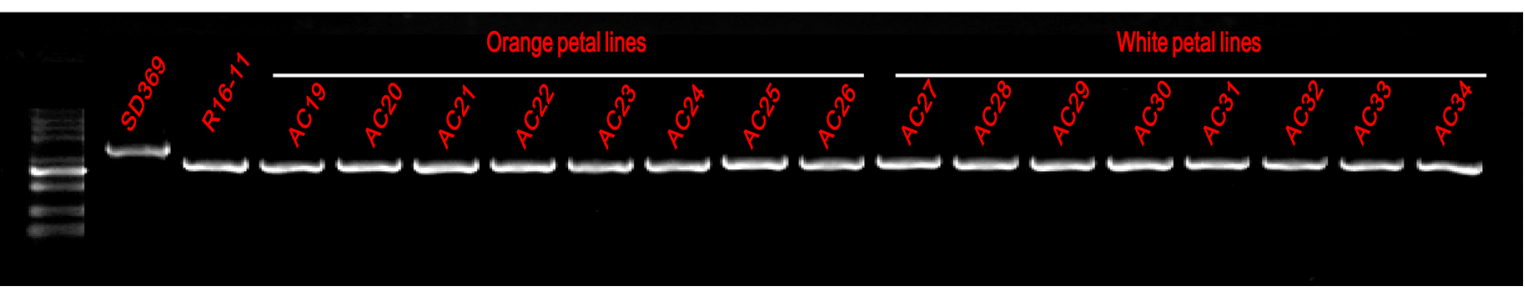

FIGURE 5 | Candidate gene analysis of Br-dyp1. (A) Br-dyp1 includes 14 exons and 13 introns in the two parents. (B) A 679-bp insertion in dark-yellow-flowered SD369 caused a premature stop codon. (C-E) Validation of the functional marker Br-dyp1-InDel in $F_{2}$ individuals (C), in 8 yellow and 8 dark-yellow-flowered materials (D), and in eight orange and eight white flowered materials (E).

component in the memory of the chloroplast with respect to preceding photo-oxidative stress (Jahns and Holzwarth, 2012). In this study, genes participating in the "response to heat (GO: 0009408)," the "response to hydrogen peroxide (GO: 0042542)," the "fructose 1,6-bisphosphate metabolic process (GO: 0030388)," and "carbohydrate transport (GO: 0008643)" were significantly enriched in the biological process category (Figure 6A), which implied that due to the increased accumulation of zeaxanthin in dark yellow petals, the photo and heat stress and reactive oxygen were removed, so the genes involved in heat and hydrogen peroxide responses were downregulated. Furthermore, due to the sustained energy dissipation by zeaxanthin, genes with function of "UDP-glycosyltransferase activity (GO: 0008194)" and "sugar transmembrane transporter activity (GO: 0051119)" were downregulated (Figure 6A). Interestingly, GO enrichment analysis of the 248 upregulated genes showed that "cell wall biogenesis (GO: 0042546)," "cell wall organization (GO: 0071555), and "xyloglucan metabolic process (GO: 0010411)" were the top three significantly enriched terms in the biological process 

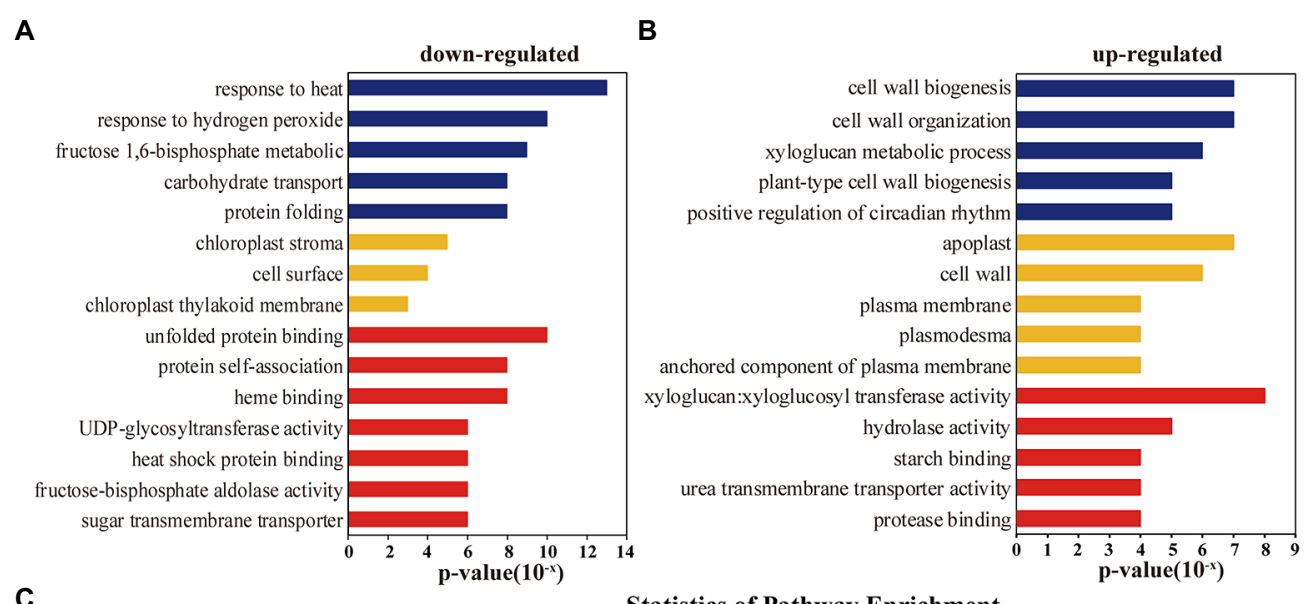

C
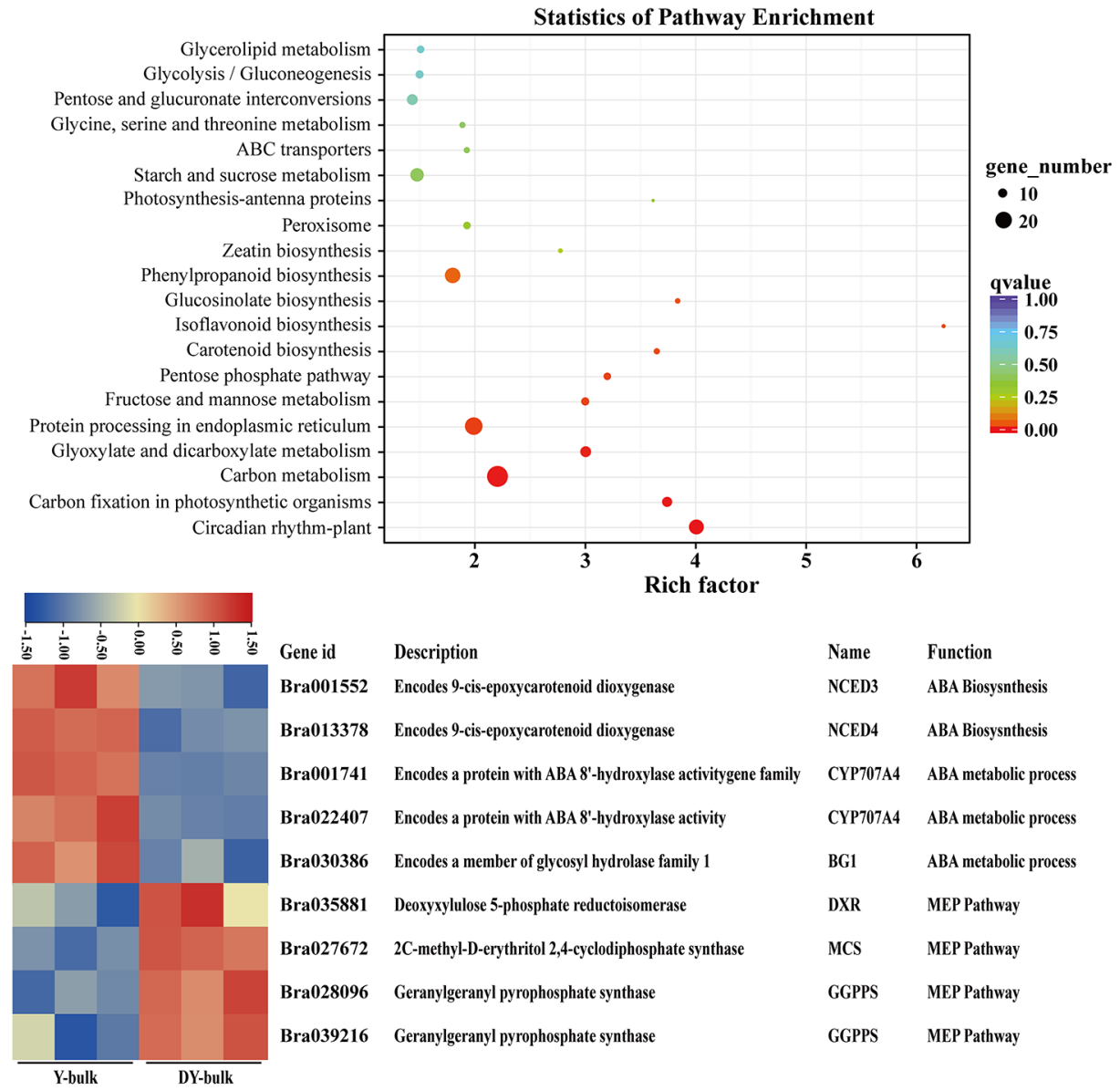

$\begin{array}{ll}\text { Name } & \text { Function } \\ \text { NCED3 } & \text { ABA Biosysnthesis } \\ \text { NCED4 } & \text { ABA Biosysnthesis } \\ \text { CYP707A4 } & \text { ABA metabolic process } \\ \text { CYP707A4 } & \text { ABA metabolic process } \\ \text { BG1 } & \text { ABA metabolic process } \\ \text { DXR } & \text { MEP Pathway } \\ \text { MCS } & \text { MEP Pathway } \\ \text { GGPPS } & \text { MEP Pathway } \\ \text { GGPPS } & \text { MEP Pathway }\end{array}$

FIGURE 6 | Transcriptome analysis in yellow and dark yellow petals. (A,B) GO terms that were significantly enriched in 587 downregulated genes (A) and in 278 upregulated genes in the DY-bulk (B). (C) Scatter plot of top 20 enriched KEGG pathways. Rich factor is the ratio of the DEG number to the background number in a certain pathway. The size of the dots represents the number of genes, and the color of the dots represents the range of the $q$-value. (D) Differentially expressed genes related to the carotenoid metabolism and flux. The heatmap colors are shown in log2 (FPKM). Three biological replicates of the W-bulk and G-bulk are shown.

category, and large proportions of DEGs were assigned to the "apoplast (GO: 0048046)" and "cell wall (GO: 0005618)" in the cellular component category (Figure 6B). Xyloglucan endotransglucosylase/hydrolase genes $(X T H s)$, which encode proteins with xyloglucan:xyloglucosyl transferase activity (GO:
0016762; Figure 6B), are involved in petal abscission in rose (Singh et al., 2011). In our study, the upregulated expression of XTHs in dark yellow petals might have caused faster petal abscission to save energy and to compensate sustained energy dissipation by zeaxanthin. 
KEGG pathway enrichment analysis revealed that "carotenoid biosynthesis" was one of the most significantly enriched pathways (Figure 6C). When focusing on specific genes, in addition to the downregulation of the candidate gene Bra037130 (BraA09. $Z E P$ ) in the DY-bulk, five genes involved in carotenoid degradation and ABA biosynthetic and metabolic processes, including NECD3 (Bra001552), NCED4 (Bra013378), CYP707A4 (Bra001741 and Bra022407) and BG1 (Bra030386; Figure 6D), were also found to be significantly downregulated (FDR $\leq 0.05$ and $\mid \log 2$ (fold change) $\mid \geq 1$ ). Notably, most genes involved in carotenoid biosynthesis, such as PSY (Bra006391), PDS (Bra010751), ZDS (Bra040411), LCYB (Bra022892 and Bra029825), and LCYE (Bra006838), were slightly downregulated (FDR $\leq 0.05$ and $\mid \log 2$ (fold change) $\mid<1$; Supplementary Table 11). However, some of the genes in the MEP pathway, such as DXR (Bra035881), MCS (Bra027672), and GGPPS (Bra028096 and Bra039216; Supplementary Table 11), were slightly upregulated, which might partially contribute to the increased total carotenoid levels. In recent years, increasing evidence of cross-talk between flavonoid and carotenoid pathways has been obtained (Davuluri et al., 2005; Meng et al., 2019; Liu et al., 2020b). In this study, most genes involved in isoflavonoids and flavonoids biosynthesis were downregulated (Supplementary Table 11), which was consistent with the previous study (Liu et al., 2020b). The "circadian rhythm" pathway was significantly enriched, and most of the genes in this pathway were downregulated (Supplementary Table 11), which concurred that the transcription of ZEP gene follows a diurnal rhythm (Audran et al., 1998; Thompson et al., 2000). Furthermore, genes in the "carbon fixation in photosynthetic organisms" pathway were significantly enriched, and most of these genes were downregulated (Supplementary Table 11), which was consistent with the results of GO enrichment analysis, suggesting that the loss-of-function of ZEP caused increased accumulation of zeaxanthin and disturbed antenna assembly and affected the photosynthesis system.

\section{DISCUSSION}

The present study successfully fine-mapped the $B r$-dyp1 gene to a physical interval of $53.6 \mathrm{~kb}$. Many lines of evidence revealed that the Bra037130 (BraA09.ZEP) gene is the candidate gene for $B r-d y p 1$. First, the functional annotation analysis of 11 genes within the $53.6 \mathrm{~kb}$ interval revealed that only one gene, Bra037130, homologous to ZEP in Arabidopsis, was involved in carotenoid biosynthesis. Second, the expression level of Bra037130 (BraA09.ZEP) in flower tissues was much lower in deep yellow petals than in yellow petals. Third, sequence alignment showed that the 679bp insertion in dark yellow petals caused a premature stop codon, thus causing the loss of function of the ZEP enzyme. Most importantly, we developed a functional marker for the candidate gene, and validation showed that this functional marker co-segregated with the petal color phenotype. Furthermore, carotenoid profile analysis showed increased accumulation of zeaxanthin and a reduction of violaxanthin, which concurred with the ZEP function (Marin et al., 1996; Gonzalez-Jorge et al., 2016). Above all, a $679 \mathrm{bp}$ insertion of Bra037130 (BraA09.ZEP) in SD369 was the main reason that caused the dark yellow petal color phenotype. The developed functional marker can be used for molecular-assisted breeding and for developing new ornamental varieties with visual appeal, which has profound significance.

The gene structure annotation of Bra037130 (BraA09.ZEP) in B. rapa V1.5 reference genome has some mistake. When we cloned the candidate gene Bra037130 (BraA09.ZEP) for $B r-d y p 1$, we first designed the primer pair Br-dyp1-F and Br-dyp1-R1 (Supplementary Table 8) to amplify the full-length sequence according to the $B$. rapa V1.5 reference genome. The results showed that no amplification products could be detected using cDNA from SD369 or R16-11 as a template. Another fragment-amplifying primer pair Br-dyp1-qF2 and Br-dyp1-qR2 (Supplementary Table 8), which targeted the eighteenth exon of Bra037130 according to the B. rapa V1.5 annotation, were designed and still no amplification products were detected using cDNA from either parent as the templates. However, the qRT-PCR results (Figure 4A) and the RNA-seq analysis (Figure 5D) showed that the candidate gene Bra037130 did express in the parental lines. Thus, there must be some mistake in the Bra037130 annotation in B. rapa V1.5. We subjected the CDS of Bra037130 to a BLAST search against the $B$. rapa V3.0 reference genome and found that the gene corresponding to Bra037130 in $B$. rapa V3.0 was BraA09g009220.3C. The BraA09g009220.3C gene owned 14 exons and 13 introns, whereas Bra037130 had 18 exons and 17 introns (Supplementary Table 12), indicating a considerable difference between the $B$. rapa V1.5 and V3.0 annotations. Therefore, we designed another full-length primer pair BrZEPful-F and BrZEP-ful-R2 to amplify the full-length sequence according to the $B$. rapa $\mathrm{V} 3.0$ reference genome, and both the full-length gDNA and cDNA could be amplified in the two parent lines (Supplementary Figure 2). Thus, the gene structure annotation of Bra037130 in B. rapa V3.0 reference genome is corrected according to our experiments results.

Through LC-APCI-MS/MS analysis with saponification, we observed that lutein was the most abundant carotenoid in yellow petals, whereas violaxanthin was the second most abundant carotenoid, accounting for approximately 57.7 and $24.1 \%$ of the total carotenoids in the Y-bulk, respectively. These results differed from our previous study (Yang et al., 2021) and another study conducted in Chinese cabbage (Zhang et al., 2020), in which the violaxanthin was the most abundant carotenoid and lutein was the second most abundant carotenoid. It has been reported that deepoxidation to zeaxanthin is favored in highlight conditions, while epoxidation to violaxanthin predominates under moderate-light conditions (Kalituho et al., 2007; GonzalezJorge et al., 2016). The reasons for the above difference might due to the different seasons and different places for the petal sample collection. Because petals were collected in November and in greenhouse in our previous study (Yang et al., 2021), in which the sunlight was mild and the epoxidation to violaxanthin was favored, whereas we collected the petals in June and in an open field for this study, where the sunlight was bright and strong, so the de-epoxidation to zeaxanthin dominated. 
A common feature of zep mutant leaves is the increased accumulation of zeaxanthin and decreased production of antheraxanthin and violaxanthin (Rock and Zeevaart, 1991; Marin et al., 1996; Niyogi et al., 1998; Liu et al., 2020b). As expected, in our study, the amount of zeaxanthin increased about 11.2-fold in DY-bulk than that in Y-bulk, and violaxanthin decreased about 3.6-fold in DY-bulk. Unexpectedly, the antheraxanthin increased about 11.5-fold in DY-bulk, which was different from the findings of previous studies (Rock and Zeevaart, 1991; Marin et al., 1996; Gonzalez-Jorge et al., 2016). We speculated that there were at least two mechanisms that might explain this difference. First, the enzyme zeaxanthin epoxidase might show substrate specificity. Phaeodactylum tricornutum contains three copies of ZEP, which exhibit different catalytic activities and substrate specificities (Eilers et al., 2016). In this study, the mutation of Br-dyp1 (BraA09. $Z E P$ ) mainly disturbed the epoxidation from antheraxanthin to violaxanthin, and the enzyme encoded by Br-dyp1 (BraA09. $Z E P$ ) might therefore exhibit substrate specificity for antheraxanthin. Second, the genome of Chinese cabbage has undergone genome triplication, and another paralogous gene, BraA07.ZEP, might contribute to epoxidation from zeaxanthin to antheraxanthin. Zeaxanthin epoxidases is present in only one gene copy in the model plants Arabidopsis or rice (Rock and Zeevaart, 1991; Agrawal et al., 2001). However, there were two copies of $Z E P$ found in Chinese cabbage, and the sequence identity between BraA07.ZEP and our candidate gene, Br-dyp1 (BraA09.ZEP), was high to $87.02 \%$ in CDS. $B r a A 07 . Z E P$ was mainly expressed in leaves, whereas $B r-d y p 1$ (BraA09.ZEP) was mainly expressed in flower tissues, the tissue-specific expression pattern showed a functional divergence, which was consistent with the results obtained in B. napus (Liu et al., 2020b). Although BraA07.ZEP was mainly expressed in leaves, its transcripts could also be detected in petals. Hence, we suspected that the enzyme encoded by BraA07.ZEP might partially compensate for the loss-of-function mutation of Br-dyp1 (BraA09.ZEP) in dark yellow petals and might be responsible for epoxidation from zeaxanthin to antheraxanthin. A transgenic line of A. thaliana with partly disabled zeaxanthin epoxidase activity also showed increased levels of zeaxanthin and antheraxanthin and decreased levels of violaxanthin (Nowicka et al., 2009), which is same with our results, and further confirming our above speculation.

In addition to the increased zeaxanthin and antheraxanthin, other xanthophylls especially the lutein increased about 6.7-fold in DY-bulk, and the total content of carotenoids showed a remarkable 4.7 -fold increase in the DY-bulk, which can also be observed in maturing Arabidopsis seeds (GonzalezJorge et al., 2016). Two possible reasons might explain this result. First, the enzyme ZEP is not only responsible for the epoxidation from zeaxanthin to violaxanthin, but may also be responsible for the epoxidation from lutein to lutein epoxide. Lutein epoxide is widespread among photosynthetic and non-photosynthetic plant tissues (Garcia-Plazaola et al., 2007), which has been detected in chromoplasts from flowers (Tai and Chen, 2000), fruits (Watanabe and Takahashi, 1999), seeds (Edelenbos et al., 2001), and tubers (Lu et al., 2001).
The loss of ZEP activity in dark yellow petals disturbed not only the xanthophyll cycle but also the lutein epoxide cycle, thus causing increases in the accumulation of zeaxanthin and lutein. Second, the mutation of the gene Br-dyp1 (BraA09. $Z E P$ ) blocked the carotenoid flux, impaired the carotenoid degradation, and disturbed the flux from carotenoid to ABA biosynthesis, thus causing the bias from the $\beta$-branch to the $\alpha$-branch, so the lutein increased and the total carotenoid increased. Our results were consistent with another study on a ZEP mutant, in which the lutein content was increased 2.2 -fold and the total seed carotenoids showed a remarkable 6 -fold increase relative to the wild type (Gonzalez-Jorge et al., 2016). In B. napus, the disruption of the gene CCD4 impairs the carotenoid degradation and disturbs the carotenoid flux and causes the total carotenoid to increase approximately 42 -fold, ultimately changing the flower color from white to yellow (Zhang et al., 2015). Finally, some genes in the MEP pathways were upregulated in the DY-bulk, while some genes for ABA biosynthesis were downregulated, which provided more precursors for carotenoid biosynthesis and alleviated the carotenoid degradation and loss, which might also contribute to the increased total carotenoids in dark yellow petals.

\section{CONCLUSION}

The present study delimited the $B r-d y p 1$ gene responsible for the dark yellow petal color trait in Chinese cabbage. The $B r-d y p 1$ gene was fine-mapped to an interval of $53.6 \mathrm{~kb}$ via BSR-Seq and linkage analysis. Through functional annotation, expression profile and sequence variation analysis, Bra037130 (BraA09.ZEP) which encodes a zeaxanthin epoxidase, was the most likely candidate gene for $B r-d y p 1$. BraA09.ZEP is involved in the epoxidation from zeaxanthin to violaxanthin. A $679 \mathrm{bp}$ insertion in dark yellow petals caused a premature stop codon, and, thus, the loss of ZEP enzyme function, which affected carotenoid metabolism and caused an increase in the accumulation of total carotenoids. Moreover, we developed and validated the functional marker Br-dyp1-InDel for $B r-d y p 1$. This achievement is an important advance for molecular research on flower pigmentation in Chinese cabbage.

\section{DATA AVAILABILITY STATEMENT}

The datasets presented in this study can be found in online repositories. The names of the repository/repositories and accession number(s) can be found in the article/ Supplementary Material.

\section{AUTHOR CONTRIBUTIONS}

$\mathrm{X}-\mathrm{WZ}$ and YY conceptualized the experiments and provided the funding resource. SY drafted the manuscript. HL and YZ performed the experiments and analyzed the data. XW, HS, 
$\mathrm{ZW}$, and $\mathrm{XZ}$ participated in drafting the article and revising it critically. All authors contributed to the article and approved the submitted version.

\section{FUNDING}

This work was financially supported by Zhongyuan Scholar Program (202101510003), the China Agriculture Research System (CARS-23-G-15), Sci-Tech Innovation Team of Henan Academy

\section{REFERENCES}

Abe, A., Kosugi, S., Yoshida, K., Natsume, S., Takagi, H., Kanzaki, H., et al. (2012). Genome sequencing reveals agronomically important loci in rice using MutMap. Nat. Biotechnol. 30, 174-178. doi: 10.1038/nbt.2095

Agrawal, G. K., Yamazaki, M., Kobayashi, M., Hirochika, R., Miyao, A., and Hirochika, H. (2001). Screening of the rice viviparous mutants generated by endogenous retrotransposon Tos 17 insertion. Tagging of a zeaxanthin epoxidase gene and a novel ostatc gene. Plant Physiol. 125, 1248-1257. doi: 10.1104/pp.125.3.1248

Alboresi, A., Dall'osto, L., Aprile, A., Carillo, P., Roncaglia, E., Cattivelli, L., et al. (2011). Reactive oxygen species and transcript analysis upon excess light treatment in wild-type Arabidopsis thaliana vs a photosensitive mutant lacking zeaxanthin and lutein. BMC Plant Biol. 11:62. doi: 10.1186/14712229-11-62

Alder, A., Jamil, M., Marzorati, M., Bruno, M., Vermathen, M., Bigler, P., et al. (2012). The path from $\beta$-carotene to carlactone, a strigolactone-like plant hormone. Science 335, 1348-1351. doi: 10.1126/science.1218094

Alexa, A., and Rahnenfuhrer, J. (2010). topGO: enrichment analysis for gene ontology. $\mathrm{R}$ package version 2.18.0.

Audran, C., Borel, C., Frey, A., Sotta, B., Meyer, C., Simonneau, T., et al. (1998). Expression studies of the zeaxanthin epoxidase gene in nicotiana plumbaginifolia. Plant Physiol. 118, 1021-1028. doi: 10.1104/pp.118. 3.1021

Davuluri, G. R., van Tuinen, A., Fraser, P. D., Manfredonia, A., Newman, R., Burgess, D., et al. (2005). Fruit-specific RNAi-mediated suppression of DET1 enhances carotenoid and flavonoid content in tomatoes. Nat. Biotechnol. 23, 890-895. doi: 10.1038/nbt1108

Edelenbos, M., Christensen, L. P., and Grevsen, K. (2001). HPLC determination of chlorophyll and carotenoid pigments in processed green pea cultivars (Pisum sativum L.). J. Agric. Food Chem. 49, 4768-4774. doi: 10.1021/ jf010569z

Eilers, U., Dietzel, L., Breitenbach, J., Buchel, C., and Sandmann, G. (2016). Identification of genes coding for functional zeaxanthin epoxidases in the diatom Phaeodactylum tricornutum. J. Plant Physiol. 192, 64-70. doi: 10.1016/j. jplph.2016.01.006

Frey, A., Boutin, J. P., Sotta, B., Mercier, R., and Marion-Poll, A. (2006). Regulation of carotenoid and $\mathrm{ABA}$ accumulation during the development and germination of Nicotiana plumbaginifolia seeds. Planta 224, 622-632. doi: $10.1007 / \mathrm{s} 00425-006-0231-2$

Gao, Z., Liu, Q., Zheng, B., and Chen, Y. (2013). Molecular characterization and primary functional analysis of PeVDE, a violaxanthin de-epoxidase gene from bamboo (Phyllostachys edulis). Plant Cell Rep. 32, 1381-1391. doi: $10.1007 / \mathrm{s} 00299-013-1450-1$

Garcia-Plazaola, J. I., Matsubara, S., and Osmond, C. B. (2007). The lutein epoxide cycle in higher plants: its relationships to other xanthophyll cycles and possible functions. Funct. Plant Biol. 34, 759-773. doi: 10.1071/ FP07095

Gonzalez-Jorge, S., Mehrshahi, P., Magallanes-Lundback, M., Lipka, A. E., Angelovici, R., Gore, M. A., et al. (2016). ZEAXANTHIN EPOXIDASE activity potentiates carotenoid degradation in maturing seed. Plant Physiol. 171, 1837-1851. doi: 10.1104/pp.16.00604

Han, F., Cui, H., Zhang, B., Liu, X., Yang, L., Zhuang, M., et al. (2019). Mapbased cloning and characterization of BoCCD4, a gene responsible for white/ of Agricultural Sciences (2021TD06), and the Self-dependent Innovation Program in Henan Academy of Agricultural Science (2121ZC23).

\section{SUPPLEMENTARY MATERIAL}

The Supplementary Material for this article can be found online at: https://www.frontiersin.org/articles/10.3389/fpls.2022.841328/ full\#supplementary-material

yellow petal color in B. oleracea. BMC Genomics 20:242. doi: 10.1186/ s12864-019-5596-2

Jahns, P., and Holzwarth, A. R. (2012). The role of the xanthophyll cycle and of lutein in photoprotection of photosystem II. Biochim. Biophys. Acta 1817, 182-193. doi: 10.1016/j.bbabio.2011.04.012

Kalituho, L., Rech, J., and Jahns, P. (2007). The roles of specific xanthophylls in light utilization. Planta 225, 423-439. doi: 10.1007/s00425-006-0356-3

Kevan, P. G., and Baker, H. G. (1983). Insects as flower visitors and pollinators. Annu. Rev. Entomol. 28, 407-453. doi: 10.1146/annurev.en.28.010183.002203

Kim, D., Langmead, B., and Salzberg, S. L. (2015). HISAT: a fast spliced aligner with low memory requirements. Nat. Methods 12, 357-360. doi: 10.1038/nmeth.3317

Kosambi, D. D. (1943). The estimation of map distances from recombination values. Ann. Eugenics 12, 172-175. doi: 10.1111/j.1469-1809.1943.tb02321.x

Li, H., and Durbin, R. (2010). Fast and accurate long-read alignment with burrows-wheeler transform. Bioinformatics 26, 589-595. doi: 10.1093/ bioinformatics/btp698

Li, H., Handsaker, B., Wysoker, A., Fennell, T., Ruan, J., Homer, N., et al. (2009). The sequence alignment/map format and SAMtools. Bioinformatics 25, 2078-2079. doi: 10.1093/bioinformatics/btp352

Li, L., Yuan, H., Zeng, Y., and Xu, Q. (2016). Plastids and carotenoid accumulation. Subcell. Biochem. 79, 273-293. doi: 10.1007/978-3-319-39126-7_10

Liu, Y., Lv, J., Liu, Z., Wang, J., Yang, B., Chen, W., et al. (2020a). Integrative analysis of metabolome and transcriptome reveals the mechanism of color formation in pepper fruit (Capsicum annuum L.). Food Chem. 306:125629. doi: 10.1016/j.foodchem.2019.125629

Liu, Y., Ye, S., Yuan, G., Ma, X., Heng, S., Yi, B., et al. (2020b). Gene silencing of BnaA09.ZEP and BnaC09.ZEP confers orange color in Brassica napus flowers. Plant J. 104, 932-949. doi: 10.1111/tpj.14970

Livak, K. J., and Schmittgen, T. D. (2001). Analysis of relative gene expression data using real-time quantitative PCR and the $2^{-\Delta \Delta C T}$ method. Methods 25 , 402-408. doi: 10.1006/meth.2001.1262

Lou, Y., Sun, H., Li, L., Zhao, H., and Gao, Z. (2017). Characterization and primary functional analysis of a bamboo ZEP gene from phyllostachys edulis. DNA Cell Biol. 36, 747-758. doi: 10.1089/dna.2017.3705

Love, M. I., Huber, W., and Anders, S. (2014). Moderated estimation of fold change and dispersion for RNA-seq data with DESeq2. Genome Biol. 15:550. doi: 10.1186/s13059-014-0550-8

Lu, W., Haynes, K., Wiley, E., and Clevidence, B. (2001). Carotenoid content and color in diploid potatoes. J. Am. Soc. Hortic. Sci. 126, 722-726. doi: 10.21273/jashs.126.6.722

Mao, X., Cai, T., Olyarchuk, J. G., and Wei, L. (2005). Automated genome annotation and pathway identification using the KEGG Orthology (KO) as a controlled vocabulary. Bioinformatics 21, 3787-3793. doi: 10.1093/ bioinformatics/bti430

Marin, E., Nussaume, L., Quesada, A., Gonneau, M., Sotta, B., Hugueney, P., et al. (1996). Molecular identification of zeaxanthin epoxidase of Nicotiana plumbaginifolia, a gene involved in abscisic acid biosynthesis and corresponding to the ABA locus of Arabidopsis thaliana. EMBO J. 15, 2331-2342. doi: 10.1002/j.1460-2075.1996.tb00589.x

Meng, Y., Wang, Z., Wang, Y., Wang, C., Zhu, B., Liu, H., et al. (2019). The MYB activator WHITE PETAL1 associates with MtTT8 and MtWD40-1 to regulate carotenoid-derived flower pigmentation in Medicago truncatula. Plant Cell 31, 2751-2767. doi: 10.1105/tpc.19.00480 
Niyogi, K. K., Grossman, A. R., and Bjorkman, O. (1998). Arabidopsis mutants define a central role for the xanthophyll cycle in the regulation of photosynthetic energy conversion. Plant Cell 10, 1121-1134. doi: 10.2307/3870716

Nowicka, B., Strzalka, W., and Strzalka, K. (2009). New transgenic line of Arabidopsis thaliana with partly disabled zeaxanthin epoxidase activity displays changed carotenoid composition, xanthophyll cycle activity and nonphotochemical quenching kinetics. J. Plant Physiol. 166, 1045-1056. doi: 10.1016/j.jplph.2008.12.010

Park, H. Y., Seok, H. Y., Park, B. K., Kim, S. H., Goh, C. H., Lee, B. H., et al. (2008). Overexpression of Arabidopsis ZEP enhances tolerance to osmotic stress. Biochem. Biophys. Res. Commun. 375, 80-85. doi: 10.1016/j. bbrc.2008.07.128

Qi, J., Yu, S., Zhang, F., Shen, X., Zhao, X., Yu, Y., et al. (2010). Reference gene selection for real-time quantitative polymerase chain reaction of mRNA transcript levels in Chinese cabbage (Brassica rapa L. ssp. pekinensis). Plant Mol. Biol. Report. 28, 597-604. doi: 10.1007/s11105-010-0185-1

Rock, C. D., Bowlby, N. R., Hoffmann-Benning, S., and Zeevaart, J. A. (1992). The aba mutant of Arabidopsis thaliana (L.) heynh. Has reduced chlorophyll fluorescence yields and reduced thylakoid stacking. Plant Physiol. 100, 1796-1801. doi: 10.1104/pp.100.4.1796

Rock, C. D., and Zeevaart, J. A. (1991). The aba mutant of Arabidopsis thaliana is impaired in epoxy-carotenoid biosynthesis. Proc. Natl. Acad. Sci. U. S. A. 88, 7496-7499. doi: 10.1073/pnas.88.17.7496

Singh, A. P., Tripathi, S. K., Nath, P., and Sane, A. P. (2011). Petal abscission in rose is associated with the differential expression of two ethylene-responsive xyloglucan endotransglucosylase/hydrolase genes, RbXTH1 and RbXTH2. J. Exp. Bot. 62, 5091-5103. doi: 10.1093/jxb/err209

Su, T., Yu, S., Zhang, J. W. F., Yu, Y., Zhang, D., Zhao, X., et al. (2014). Loss of function of the carotenoid Isomerase gene BrCRTISO confers Orange color to the inner leaves of Chinese cabbage (Brassica rapa L. ssp. pekinensis). Plant Mol. Biol. Report. 33, 648-659. doi: 10.1007/s11105-014-0779-0

Tai, C. Y., and Chen, B. H. (2000). Analysis and stability of carotenoids in the flowers of daylily (Hemerocallis disticha) as affected by various treatments. J. Agric. Food Chem. 48, 5962-5968. doi: 10.1021/jf000956t

Takagi, H., Abe, A., Yoshida, K., Kosugi, S., Natsume, S., Mitsuoka, C., et al. (2013). QTL-seq: rapid mapping of quantitative trait loci in rice by whole genome resequencing of DNA from two bulked populations. Plant J. 74, 174-183. doi: 10.1111/tpj.12105

Thompson, A. J., Jackson, A. C., Parker, R. A., Morpeth, D. R., Burbidge, A., and Taylor, I. B. (2000). Abscisic acid biosynthesis in tomato: regulation of zeaxanthin epoxidase and 9-cis-epoxycarotenoid dioxygenase mRNAs by light/dark cycles, water stress and abscisic acid. Plant Mol. Biol. 42, 833-845. doi: 10.1023/a:1006448428401

Van Ooijen, J.W. (2006). JoinMap 4: Software for the Calculation of Genetic Linkage Maps in Experimental Populations of Diploid Species. Wageningen, Netherlands: Plant Research International BV and Kayazma BV.

Walter, M. H., and Strack, D. (2011). Carotenoids and their cleavage products: biosynthesis and functions. Nat. Prod. Rep. 28, 663-692. doi: 10.1039/ c0np00036a

Wang, H., Huang, Y., Xiao, Q., Huang, X., Li, C., Gao, X., et al. (2020). Carotenoids modulate kernel texture in maize by influencing amyloplast envelope integrity. Nat. Commun. 11:5346. doi: 10.1038/s41467-020-19196-9
Watanabe, K., and Takahashi, B. (1999). Chlorophyll and carotenoid pigments in green- and yellow-fleshed kiwifruit during fruit development and storage. J. Japan. Soc. Hortic. sci. 68, 1038-1043. doi: 10.2503/jjshs.68.1038

Wu, J., Mao, X., Cai, T., Luo, J., and Wei, L. (2006). KOBAS server: a webbased platform for automated annotation and pathway identification. Nucleic Acids Res. 34, W720-W724. doi: 10.1093/nar/gkl167

Xiong, L., Lee, H., Ishitani, M., and Zhu, J. K. (2002). Regulation of osmotic stress-responsive gene expression by the LOS6/ABA1 locus in Arabidopsis. J. Biol. Chem. 277, 8588-8596. doi: 10.1074/jbc.M109275200

Yang, S., Tian, X., Wang, Z., Wei, X., Zhao, Y., Su, H., et al. (2021). Fine mapping and candidate gene identification of a white flower gene BrWF3 in Chinese cabbage (Brassica rapa L. ssp. pekinensis). Front. Plant Sci. 12:646222. doi: 10.3389/fpls.2021.646222

Yang, S., Yu, W., Wei, X., Wang, Z., Zhao, Y., Zhao, X., et al. (2020). An extended KASP-SNP resource for molecular breeding in Chinese cabbage (Brassica rapa L. ssp. pekinensis). PLoS One 15:e0240042. doi: 10.1371/journal.pone.0240042

Zhang, N., Chen, L., Ma, S., Wang, R., He, Q., Tian, M., et al. (2020). Fine mapping and candidate gene analysis of the white flower gene Brwf in Chinese cabbage (Brassica rapa L.). Sci. Rep. 10:6080. doi: 10.1038/ s41598-020-63165-7

Zhang, X., Li, R., Chen, L., Niu, S., Chen, L., Gao, J., et al. (2018a). Finemapping and candidate gene analysis of the Brassica juncea white-flowered mutant Bjpc2 using the whole-genome resequencing. Mol. Gen. Genomics. 293, 359-370. doi: 10.1007/s00438-017-1390-5

Zhang, X., Li, R., Chen, L., Niu, S., Li, Q., Xu, K., et al. (2018b). Inheritance and gene mapping of the white flower trait in Brassica juncea. Mol. Breed. 38:20. doi: 10.1007/s11032-017-0771-0

Zhang, B., Liu, C., Wang, Y., Yao, X., Wang, F., Wu, J., et al. (2015). Disruption of a CAROTENOID CLEAVAGE DIOXYGENASE 4 gene converts flower colour from white to yellow in brassica species. New Phytol. 206, 1513-1526. doi: 10.1111/nph.13335

Zhou, W., Niu, Y., Ding, X., Zhao, S., Li, Y., Fan, G., et al. (2020). Analysis of carotenoid content and diversity in apricots (Prunus armeniaca L.) grown in China. Food Chem. 330:127223. doi: 10.1016/j.foodchem.2020.127223

Conflict of Interest: The authors declare that the research was conducted in the absence of any commercial or financial relationships that could be construed as a potential conflict of interest.

Publisher's Note: All claims expressed in this article are solely those of the authors and do not necessarily represent those of their affiliated organizations, or those of the publisher, the editors and the reviewers. Any product that may be evaluated in this article, or claim that may be made by its manufacturer, is not guaranteed or endorsed by the publisher.

Copyright (c) 2022 Yang, Liu, Zhao, Su, Wei, Wang, Zhao, Zhang and Yuan. This is an open-access article distributed under the terms of the Creative Commons Attribution License (CC BY). The use, distribution or reproduction in other forums is permitted, provided the original author(s) and the copyright owner(s) are credited and that the original publication in this journal is cited, in accordance with accepted academic practice. No use, distribution or reproduction is permitted which does not comply with these terms. 